\title{
Performance of portable handheld ultrasound system in fetal therapy
}

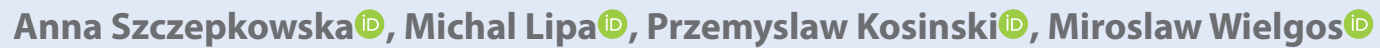 \\ $1^{\text {st }}$ Department of Obstetrics and Gynecology, Medical University of Warsaw, Poland
}

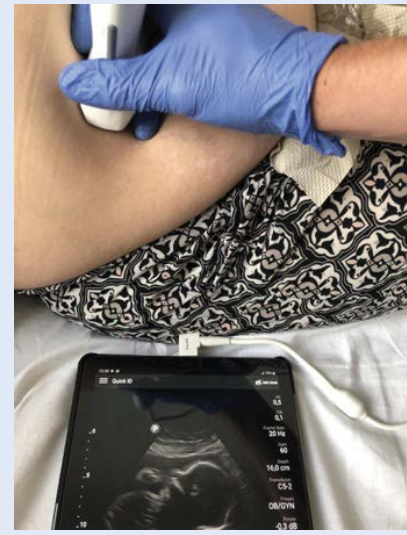

Figure 1. Portable ultrasound system in use

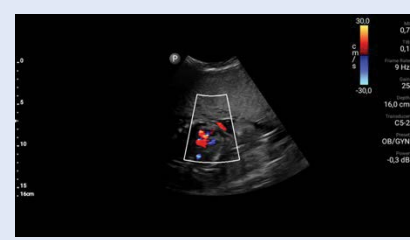
chest revealing fetal heart rate after intravenous blood transfusion and severe placentomegaly

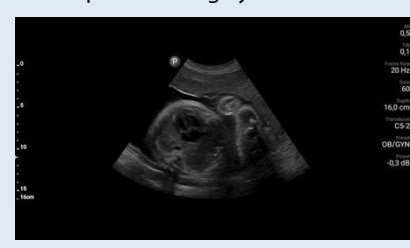

Figure 3. Cross section of fetal thorax after shunt placement
Figure 2. Cross section of the fetal

There is no doubt ultrasound remains the most important, safe, non-invasive and acceptable diagnostic method in obstetrics and gynecology. Throughout the years, new technological advances have significantly improved imaging quality, prenatal detection of congenital defects and ultrasonographic assistance in the field of fetal therapy [1]. Easy access allows more direct investigation and fetal wellbeing monitoring, especially in cases of emergency events [1]. Certainly, portable ultrasound systems open new opportunities in the fetal therapy, especially during preparation of the intrauterine procedures, ultrasonographic guidance and fetal monitoring after intrauterine surgeries/ invasive procedures [1]. In this paper, we present several, clinical applications of portable ultrasound system (Fig. 1) in patients who have undergone fetal therapy.

Careful planning is essential in fetal therapy- invasive procedures require proper precision and accuracy. This can be achieved even before the use of a regular high-resolution ultrasound machine to assess placental location and fetal position to plan the incision site. Portable ultrasound devices are even more useful in early follow-up after invasive procedures [1]. Normal fetal heart rate after prenatal intervention displayed on the monitor remains an especially important psychological aspect for patients and good prognostic factor for further pregnancy outcome.

Twin-to-twin transfusion syndrome (TTTS), a severe complication that affects about $10 \%$ of monochorionic twin pregnancies, can be effectively treated with endoscopic laser coagulation of the vascular anastomoses present on the fetal side of the placenta [2]. Endoscopic laser coagulation may be also used as a treatment in twin reversed arterial perfusion syndrome (TRAP) to occlude umbilical vessels in the acardiac twin [3]. As the first several days after the laser procedures in twins seem to determine long-term outcomes, serial monitoring of fetal heart rates, deepest vertical pockets (DVP) of amniotic fluids and appearance of fetal bladders remain the most important aspects in postsurgical monitoring. As patients may feel uncomfortable after these procedures, portable ultrasound systems may help to monitor hemodynamic, fetal status on the beside with no need to refer patient to the ultrasound department.

Rhesus disease or infections with Parvovirus B19 may induce progressing fetal anemia, heart failure or intrauterine death. The gold standard of diagnosis and treatment of these pathologies are intrauterine blood transfusions. The technique, injection site and volume of transfused blood depend on gestational age and hemodynamic status. In cases of severe anemia usually fetal hydrops coexist. Serial blood transfusions may prevent from cardiac failure and adverse pregnancy outcome [4]. In this group of patients, portable ultrasound allows to register fetal heart rate following the procedure. Figure 2 shows fetus with severe hydrops fetalis and severe anemia due to Parvovirus B19 infection few hours after blood transfusion.

In cases of pleural effusion ultrasound-guided placement of thoraco-amniotic shunt can bring benefits such as restoring normal intrathoracic anatomy, reducing the risk of lung hypoplasia.

Portable system can be useful in clinical setting after the procedure to confirm correct shunt's placement (Fig. 3) and fetal heart rate, particularly during ward rounds and when a patient reports complaint. It also allows easy evaluation of pleural effusion and amniotic fluid index.

Hand-held ultrasound devices are easy and convenient for both, physicians and patients [1]. As an ultrasound scan is possible instantly at a patient's bedside it is useful in emergency situations. Considering the abovementioned, clinical applications it seems that portable ultrasound systems may be widely used in the future, not only in fetal surgery, but in other fields as well.

Corresponding author:

Przemyslaw Kosinski

$1^{\text {st }}$ Department of Obstetrics and Gynecology, Medical University of Warsaw, Poland, 1/3 Sokratesa St, 02-015 Warsaw, Poland

e-mail: pkosinski@wum.edu.pl 


\section{REFERENCES:}

1. Rykkje A, Carlsen JF, Nielsen MB. Hand-Held Ultrasound Devices Compared with High-End Ultrasound Systems: A Systematic Review. Diagnostics (Basel). 2019; 9(2), doi: 10.3390/diagnostics9020061, indexed in Pubmed: 31208078.

2. Bolch C, Fahey M, Reddihough D, et al. Twin-to-twin transfusion syndrome neurodevelopmental follow-up study (neurodevelopmental outcomes for children whose twin-to-twin transfusion syndrome was treated with placental laser photocoagulation). BMC Pediatr. 2018; 18(1): 256, doi: 10.1186/s12887-018-1230-8, indexed in Pubmed: 30068295.

3. Buyukkaya A, Tekbas G, Buyukkaya R. Twin Reversed Arterial Perfusion (TRAP) Sequence; Characteristic Gray-Scale and Doppler Ultrasonography Findings. Iranian Journal of Radiology. 2015; 12(3), doi: 10.5812/iranjradiol.12(3)2015.14979.

4. Jong Ede, Haan Tde, Kroes A, et al. Parvovirus B19 infection in pregnancy. Journal of Clinical Virology. 2006; 36(1): 1-7, doi: 10.1016/j.jcv.2006.01.004. 